\title{
A Method of Multi-License Plate Location in Road Bayonet Image
}

\author{
Ying Qian \\ The lab of Graphics and Multimedia \\ Chongqing University of Posts and Telecommunications \\ Chongqing, China
}

\begin{abstract}
To solve the problem of multi-license plate location in road bayonet image, a novel approach was presented, which utilized plate's color features, geometry characteristics and gray feature. Firstly, the RGB color image was converted to HSV color model and calculates the distance according to the plate's color information in the color space. Secondly, the license plate candidate regions were segmented by binary and morphological processing. Finally, based on the plate's geometry characteristics and gray feature, the license plate regions were segmented by and validated. In a certain degree, the method wasn't limited the plate's type, size, number, the location of the car and the background in the picture. It was tested using the road bayonet image.(Abstract)
\end{abstract}

Keywords-multi-license plate location; color features; geometry characteristics; gray feature

\section{INTRODUCTION}

At present, the intelligent transportation system commonly used HD intelligent traffic cameras, which has a wide range of monitoring. The system can capture two or three vehicle lanes by using HD traffic cameras, which has significantly improved the efficiency. At the same time, the equipment cost and maintenance cost has been saved. The license plate recognition system is mainly composed of license plate location, character segmentation and character recognition. Among them, the license plate location is the premise and foundation of license plate character segmentation and character recognition.

There are many kinds of method for license plate location, but most methods aiming at the single license plate or the plate in the semi-structure environment, such as charging stations, small import and export. Which has constrained in the application, such as requiring the plate size and position in the image varies in a certain range. Jie Guo et al [1] convert the image from RGB color space to HSV color space and then segment the regions which satisfied the color feature of plate by calculating the distance and similarity in color space. To the segmented image, the texture and structural features are analyzed to locate the license plate correctly. De-hua Ren [2] proposed a color classification method based on the distance between different colors according to the Chinese car license plate color features and then segmented the license-plate's background color regions by scanning lines of picture and analyzing the line segments. Finally, these regions were translated into binary image in which license-plate's background color was dark and license-plate's foreground color was white, and validated by the license-plate's gray

\author{
Zhi Li \\ The lab of Graphics and Multimedia \\ Chongqing University of Posts and Telecommunications \\ Chongqing, China
}

features. The two methods could adapt to license-plate's type, size, number and weren't limited to the location of the car and the background in the image. But in the application of the multi-license plate location in road bayonet image, as a result of multiple lanes, the disturbance of the trees, billboards and the reason of license plate dirty, wear hardly, the above methods can't locate all regions of license plate in the image.

This paper aimed at the problem of multi-license plate location in road bayonet image, firstly calculates the distance according to the plate's color features in HSV color space and then segments the regions of interest by binary and morphological processing. Finally, based on the plate's geometry characteristics and gray feature, the license plate regions were segmented and validated. The method combines the license-plate's color features, geometry characteristics and gray feature, which can locate all regions of license plate in road bayonet image.

\section{COLOR IMAGE PREPROCESSING}

\section{A. Color models}

According to different applications, color representation in different ways. RGB color model is used for display, TV and scanner device, use three basic colors of red, blue and green configures most of the color which human eye can see. The HSV color model is widely used in video and television broadcasting. H,S,V respectively represent the Hue, Saturation and Value, which corresponding with the color features of the human eye can perceive. This color model represented by Munsell three-dimensional space coordinate system, because of the psychological perception of independence between the coordinates, it can independently perceive the change of color components, and because of the color is linear scalability, which suitable for user judgment with the naked eye. At the same time as the HSV model corresponds to the painter of color model, which can reflect the human perception and discrimination to the color and suitable for similarity comparison of color image [1], so this paper used the HSV color space to segment the color image.

Because of the image generally use the RGB model, so the first thing is conversion. The relationship of each component between the HSV color model and the RGB color model is as follows [3]:

$$
\max =\max \{r, g, b\}, \min =\min \{r, g, b\}
$$




$$
\begin{aligned}
& H= \begin{cases}0^{\circ} & \max =\min \\
60^{\circ} \times \frac{g-b}{\max -\min }+0^{\circ} & \max =r, g \geq b \\
60^{\circ} \times \frac{g-b}{\max -\min }+360^{\circ} & \max =r, g<b \\
60^{\circ} \times \frac{b-r}{\max -\min }+120^{\circ} & \max =g \\
60^{\circ} \times \frac{r-g}{\max -\min }+240^{\circ} & \max =b\end{cases} \\
& S=\left\{\begin{array}{cc}
0 \quad \max =0 & \\
\frac{\max -\min }{\max } \quad \max \neq 0 &
\end{array}\right. \\
& V=\max
\end{aligned}
$$

Among them, Hue with the metric, range from $0^{\circ}$ to $360^{\circ}$, Saturation values range from 0 to 1 , Value range from 0 to 1 .

\section{B. The distance in color space}

License plate background and character color of Chinese has a fixed collocation, mainly contain blue background with white characters, yellow background with black characters, white background with black or red characters and black background with white characters. The three components of $\mathrm{R}, \mathrm{G}, \mathrm{B}$ in values equal to 0 and 255 consisting of eight kinds of basic color [3], this paper selects four base color, which is associated with the plate background were blue, yellow, white and black. The RGB values of four base colors and the corresponding HSV values is shown in Table 1.

In HSV color space, the distance between $C_{1}=\left(h_{1}, s_{1}, v_{1}\right)$ and $C_{2}=\left(h_{2}, s_{2}, v_{2}\right)$ is as follows:

$$
\begin{gathered}
d\left(C_{1}, C_{2}\right)=\left[\left(v_{1}-v_{2}\right)^{2}+\left(s_{1} \cdot \cos h_{1}-s_{2} \cdot \cos h_{2}\right)^{2}+\right. \\
\left.\left(s_{1} \cdot \sin h_{1}-s_{2} \cdot \sin h_{2}\right)^{2}\right]^{\frac{1}{2}}
\end{gathered}
$$

Respectively calculated the distance from each pixel in HSV image to four base colors can obtain the feature image based on color features. The smaller the value of the pixel in feature image is, the color of the pixel in RGB space is more close to the base color.

TABLE I. RGB AND HSV VALUES OF FOUR BASE COLORS

\begin{tabular}{|l|l|l|l|l|}
\hline \multirow{2}{*}{ Color space } & \multicolumn{4}{|l|}{ Four base colors } \\
\cline { 2 - 5 } & Blue & Yellow & White & Black \\
\hline \multirow{2}{*}{ RGB } & $0,0,255$ & $255,255,0$ & $255,255,255$ & $0,0,0$ \\
\hline HSV & $240,1,1$ & $60,1,1$ & $0,0,1$ & $0,0,0$ \\
\hline
\end{tabular}

\section{Binarization of feature image}

In order to further separate the license plate from complex background, also need convert the feature image into binary image. Before the binary processing should find the appropriate threshold $T$, usually the OTSU is the ideal method to obtain the threshold, but in this paper, due to the license plate region occupied small proportion in the image and presence of large area of blue or yellow interference region, the binary image obtained from OTSU method usually contains lots of independent information.

Found in the course of the experiment, the total area of the license plate region in the image in a certain range and the value of license plate area in feature image is small. Decreased the value of the threshold obtained from OTSU method, the number of white pixels reduced present certain rules in binary image.

The following describes specific steps to obtain appropriate threshold $T$ :

1) Calculate the number of the white pixels in binary image through the OTSU method.

2) Decrease the threshold obtained from OTSU method, then calculate the reduce number of white pixels and the total number of white pixels in binary image which processed by the reduced threshold.

3) If the reduce number or the total number satisfied the rules obtained by experiment debugging, $T$ is equal to the reduced threshold. Otherwise returns step 2).

The binary image converted from feature image by appropriate threshold $T$ is as shown in Fig.1(b).
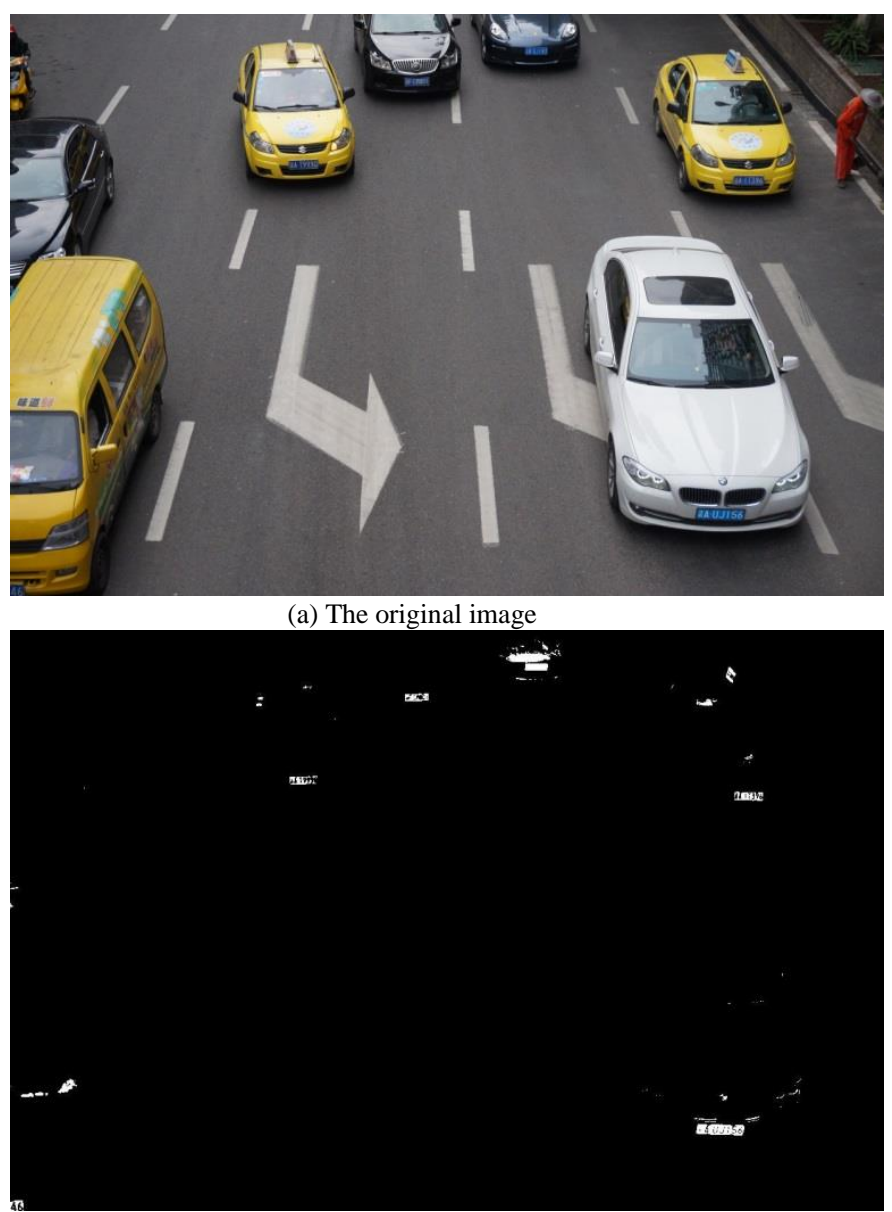

(b) The binary image

Fig. 1. The original image and the binary image

\section{Morphology operation}

Although the binary processing has filter out most background information of the image, there are still some noise and vehicle information. Therefore, mathematic morphologic close and open operation are used to make the possible license plate region into the rectangular connected region. 
The selection of structure element associated with the size of the license plate in road bayonet image, so set the close operation structure element in the image of three lanes is $5 \times 10$ and the structure element in four lanes image is $3 \times 7$. In order to remove the isolated points and smooth edge, set the open structure element is $2 \times 2$. The morphological image is as shown in Fig.2.

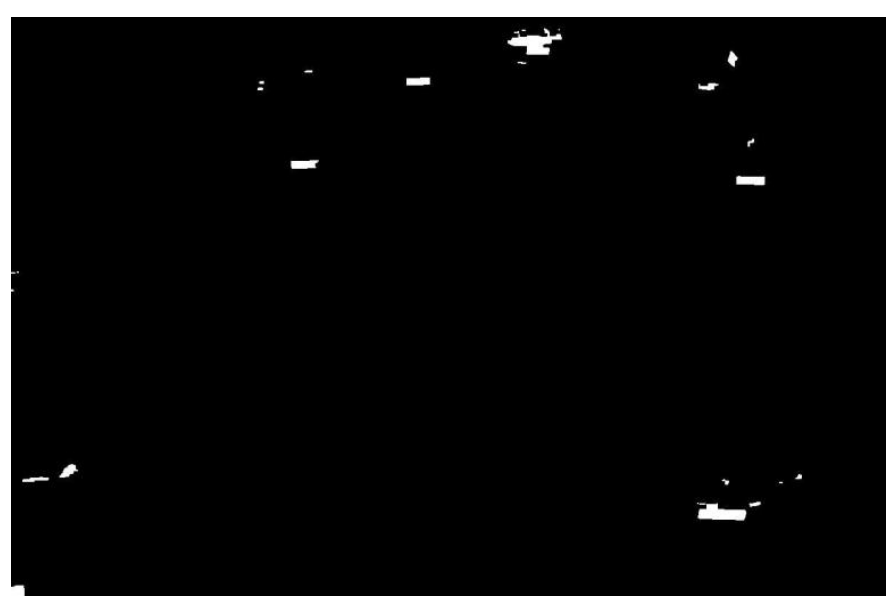

Fig. 2. The morphological image

\section{E. Connected componet labeling}

After morphology operation we can obtain the independent connected domain by 8 - connected component labeling, save the results in $L(x, y)$ and the number as $N$. the process is as follows:

$$
L(x, y)= \begin{cases}i & 1 \leq i \leq N \\ 0 & \text { other }\end{cases}
$$

The connected domain is expressed as the candidate license plate region.

\section{LOCATION OF LiCENSE PlATE}

\section{A. Geometry characteristics of license plate}

License plate has obvious geometry characteristics, the width and height of plate are fixed and the ratio of width and height is in a certain range. In China, the ratio of small car license plate is 3.14 and the ratio of large car is 2 . Considering the road bayonet image obtained from fixed traffic camera, the license plate size in the image is in a certain range and related to the size of the image. So calculate three characteristic values of the candidate license plate region, which are size, ratio and filling.

\section{B. Location of candidate license plate}

The connected domain is expressed as the candidate license plate region, so the size of the region is the area of the connected domain and the ratio is the ratio of the ratio of the minimum enclosing rectangle. The filling defined as follows:

$$
P=\frac{\text { area of connected domain }}{\text { area of minimum enclosing rectangle }}
$$

The following describes specific steps to locate license plate:

1) Set size range from $S_{\min }$ to $S_{\max }$ obtained by experiment, the connected domain which satisfied the range for the next step.

2) Conserding the reason of shooting angle and tilt, set the ratio range from 2 to 6 .

3) The $P$ is closer to 1 , it means that the connected domain is closer to the license plate. Considering the deletion of part plate information in binary and morphology operation, set the $P$ range from 0.6 to 1 .

4) Segment the region satisfied all of the conditions mentioned above in the original image and eliminate satisfied regions and small regions in the morphological image.

\section{Secondary location of candidate license plate}

The regions exist in morphological image after location include the large area regions (include license plate or not) and the region disturbed by solitary lines or vehicle information. So use the secondary location of candidate regions to locate the rest of license plate in the image. The following describes specific steps:

1) Obtained the coordinates of the current connected domain and then segmented the region in the feature image.

2) Decrease the threshold $T$, obtained the candidate region through binary processing and morphology operation.

3) Segment the regions which satisfied the conditions mentioned in location of candidate license plate in the original image.

The result of the location of candidate license plate is shown in Fig.3 and the license plate segmented from the original image is shown in Fig.4. There are two false results.

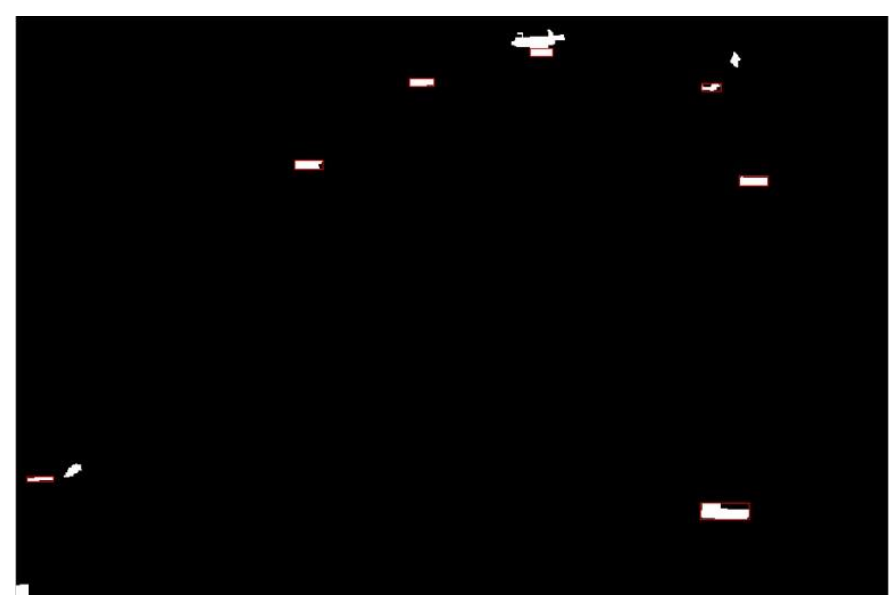

Fig. 3. The results of location in morpological image(red line marking the license plate region) 


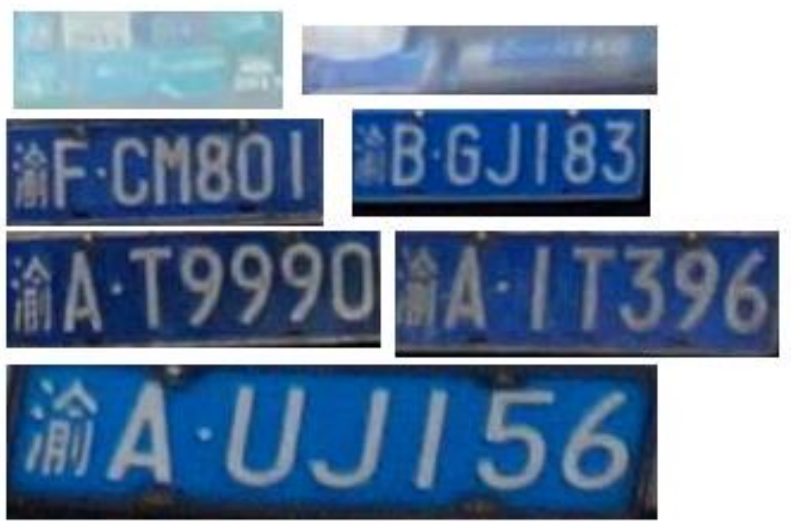

Fig. 4. The results of segmentation in the original image

\section{Validation of license plate}

The result of the license plate segmented from the original image may include some false license plate, so we validated the result based on the gray feature of license plate. The following describes specific steps:

1) Obtained the binary image of the color license plate segmented form original image by OTSU method.

2) Get the middle $80 \%$ of the binary image to remove the interference of the border in vertical projection.

3) Count the changing times of the character and the background, remove the result which unsatisfied with the gary feature.

The process of validation of license plate is shown in Fig.5.

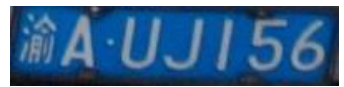

(1)License plate image

\section{涌A UJI56}

(2)License plate image

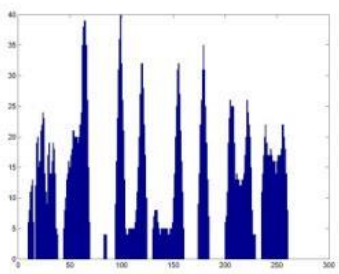

(3)Vertical projection

(a)Validation of license plate

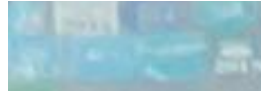

(1) False result

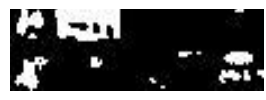

(2) False result

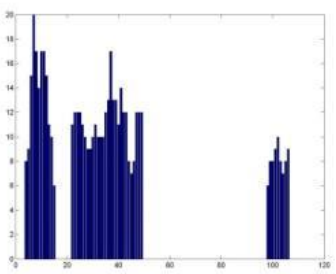

(3) Vertical projection

(b) Validation of false result
Fig. 5. The process of validation of license plate (a)(1) is the right color license plate image, (a)(2) is the binary image of the right license plate, (a)(3) is the vertical projection image of license plate. (b)(1) is the false plate image, (b)(2) is the binary image of false plate image, (b)(3) is the vertical projection image of false result

\section{EXPERIMENT RESULTS}

Considering the size of road bayonet image is $4912 \times 3264$, so at first we compress the size of image into $1228 \times 816$ to reduce the calculation time. The experiment shows that the method could locate all license plates in the road bayonet image. The part of experiment results are shown in Fig.6.

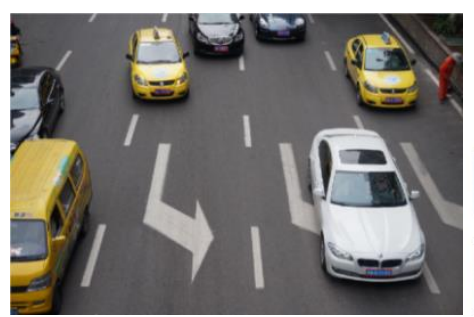

(1)The original image

(a)The location of four 1

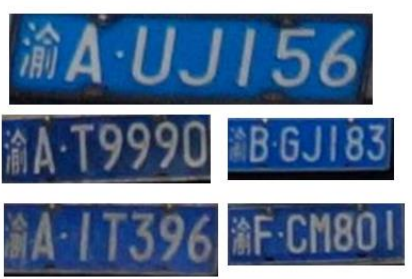

(2)The results of segmentation

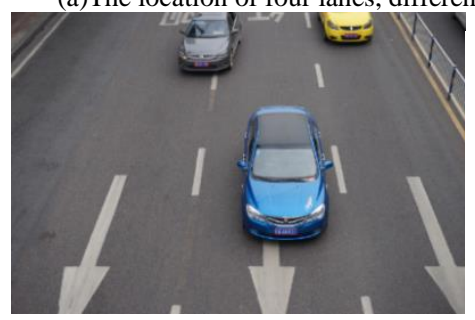

(1)The original image

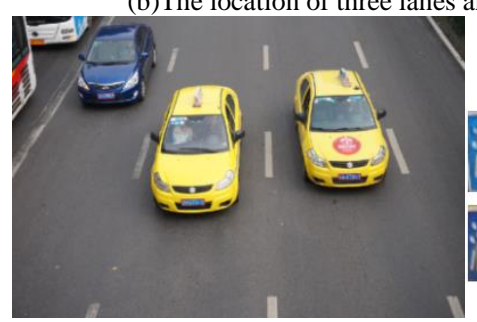

(1)The original image

\section{IIIA.6NY3!

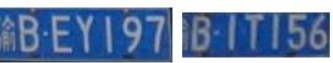

(2)The results of segmentation s and blue plate with blue car

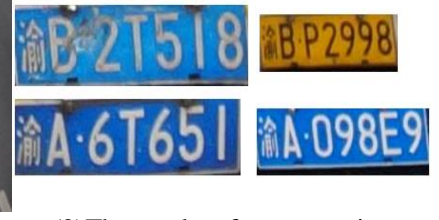

(2)The results of segmentation
Fig. 6. Part of experiment results

\section{CONCLUSIONS}

This paper aimed at the problem of multi-license plate location in road bayonet image, proposed a method combines color features, geometry characteristics and gray feature of license plate. Firstly based on the color features of license plate, filter the most background information by calculating the distance in HSV color space and binary processing. Obtained the candidate license plate regions through the morphology operation and then based on the geometry characteristics of size, ratio and filling, locate all license plate in the image by secondary location. Finally validate the results of segmentation based on the gray feature of license plate and remove the false results. This method can locate the license plate in different position, size, number and direction in the road bayonet image, which is a method of adaptability.

\section{REFERENCES}

[1] GuoJie, Shi Peng-fei. Color and texture analysis based vehicle license plate location [J]. Journal of Image and Graphics, 2002,7(5):472-476.

[2] Ren De-hua. Multi-license plate extraction based on color features in nature complex environment $[\mathrm{J}]$. Journal of Image and Graphics, 2009,14(12):2517-2526.

[3] Makoto Miyahara, Yasuhiro Yoshida. Mathematical transform of (R,G,B) color data to Munsell(H,V,C) color data [A]. In: Proceeding of SPIE Conference on Visual Communications and Image Processing [C]. Cambridge, MA, USA, 1988: 650-657. 
[4] ZhengChengyong. A novel license plate location method on RGB color space [J]. Journal of Image and Graphics, 2010,15(11):1623-1628.

[5] GuoTianshu. A car plate location method based on itself's structural features [J]. Computer \& Information Technology, 2008,10:51-57.

[6] Tan Siting, Hu Zhikun. An effective integration method for license plate location based on HSV color space [J]. Computers and Applied Chemistry, 2011,28(7):903-906.
[7] Li Wen-Ju, Liang De-qun, Zhang Qi, Fan Xin. A novel approach for vehicle license plate location based on edge-color pair [J]. Chinese Journal of Computers, 2004,27(2):204-208.

[8] Gan Ling, Sun Bo. Multiple license plate location based on separation projective and morphology operation [J]. Application Research of Computers, 2010, 29(7) : 2730-2732. 